\title{
Advanced Hodgkin's lymphoma: End-of-treatment FDG-PET should be maintained
}

\author{
Elif Hindié $^{1}$ - Charles Mesguich ${ }^{1} \cdot$ Krimo Bouabdallah $^{2} \cdot$ Noël Milpied $^{2}$
}

Published online: 2 May 2017

(C) Springer-Verlag Berlin Heidelberg 2017

Positron emission tomography with ${ }^{18} \mathrm{~F}$-fluorodeoxyglucose (FDG-PET) has become a cornerstone procedure in the modern management of Hodgkin's lymphoma (HL) [1-4]. It offers better initial staging and risk stratification [3]. FDG-PET often detects occult extranodal disease, notably focal bone marrow lesions [5-7]. It rendered useless routine bone marrow biopsy [3]. HL is associated with a high rate of residual masses at the end of therapy, which are difficult to characterize as residual disease or not on conventional imaging. Therefore, a major milestone was the finding that end-of-treatment FDG-PET offers excellent prediction of progression-free-survival (PFS), even in the presence of a residual mass [8-11]. This finding led to the introduction of FDG-PET imaging in response criteria for HL [12].

More recently, FDG-PET performed early during chemotherapy (interim-PET) has been used to tailor treatment. Here, we comment on results of two recently published prospective multicentre trials in which patients with advanced HL had response-adapted therapy based on interim-PET [13, 14]. These trials were triggered by observational studies showing high prognostic accuracy of interim PET [15]. For example, in one retrospective multicenter study of patients with advancedstage or early unfavorable HL, the 3-year PFS for patients with positive interim-PET after two cycles of ABVD (score $\geq 4$ on the Deauville 5-point scale) was $28 \%$ and for patients with negative interim-PET 95.0\% $(P<.0001)[16]$.

Elif Hindié

elif.hindie@chu-bordeaux.fr

1 Department of Nuclear Medicine, Bordeaux University Hospital, Bordeaux, France

2 Department of Hematological Oncology, Bordeaux University Hospital, Bordeaux, France
In the Southwest Oncology Group (SWOG) S0816 phase II study, which included stages III and IV HL, interim PETpositive patients after two cycles of ABVD (doxorubicin, bleomycin, vinblastine and dacarbazine) were switched to escalated-BEACOPP (bleomycin, etoposide, doxorubicin, cyclophosphamide, vincristine, procarbazine and prednisone). The 2-year PFS in interim PET-positive patients was $64 \%$ [13]. Again, in the RATHL study (stage II unfavorable, stage III and stage IV), the 3-year PFS in interim PETpositive patients who were switched from ABVD to a BEACOPP regimen was $67.5 \%$ [14]. These results are very encouraging as they show that interim PET allows a finely tuned treatment by restricting the use of BEACOPP to the $15 \%$ to $20 \%$ of patients who are PET-positive. BEACOPP offers higher cure rates in HL, but is also associated with a higher percentage of short-term and long-term adverse effects (hematologic toxicity, infections, infertility, secondary malignancies) [17]. In the absence of randomisation, the extent of benefit from switching to BEACOPP cannot be assessed from these trials [13, 14]. Also, the optimal strategy in these interim-PET-positive patients might evolve in light of the new therapeutic arsenal (targeted therapy, checkpoint inhibitors such as anti-PD-1 therapy, etc.) that is now available; for example, the possibilities of combining chemotherapy and targeted therapy with the anti-CD30 antibody-drug conjugate brentuximab vedotin [18].

Surprisingly, in both trials, the relapse rates in patients with a negative interim-PET were much higher than the rates expected from historical data [16]. In the SWOG S0816 study, which included only advanced stages, the 2-year PFS in interim PET-negative patients was $82 \%$ [13]. In the RATHL study, in which $42 \%$ of patients had stage-II disease, the 3 -year PFS in interim PET-negative patients who continued on ABVD was $85.7 \%$ [14]. The outcome was influenced by initial riskgroup: $3 y-P F S$ was $90 \%$ in stage II patients with adverse risk, 
$83.1 \%$ in stage III and $79.6 \%$ in stage IV patients [14]. Also, the risk of failure was higher in patients with IPS (international prognostic score) $\geq 3$ (3y-PFS of $81.7 \%$ ) as compared to patients with IPS $<3$ (3y-PFS of 86.9\%) [14]. These results were obtained despite the use of the standardized interpretation criteria based on the 5-point scale [3], and central reviewing of PET findings $[13,14]$. These relapse rates in interim-PETnegative patients are three times higher than expected from early reports (3-year PFS 95\%) [16] and call for maintaining a FDG PET evaluation at the end of the treatment in order to identify those patients who require further therapy. However, because the risk of relapse in interim PET negative patients varied according to initial risk stratification [14], the debate remains open, as regards cost-effectiveness, if end-oftreatment PET should be performed in all patients with advanced HL or only those with stage III-IV disease.

The yield from end-of-treatment PET in patients with negative interim-PET is difficult to assess from the published literature as most studies were performed before interim PET interpretation was standardized with Deauville criteria [19-21]. Although some authors suggested that end-oftreatment PET might not be necessary when interim-PET results are negative [20], other results point to the contrary [21]. In the study by Zinzani and colleagues, there were 157 patients with advanced HL (57 stage IIB, 62 stage III and 38 stage IV). Interim PET was negative in 123 patients; among them, ten had a positive end-of-treatment evaluation [21]. More recently, in a retrospective analysis of 76 unselected patients with HL, we showed that eight patients with negative interim-PET (Deauville criteria) had treatment failure and six of these patients were identified as non-responders at end-oftreatment PET [22]. All six patients had no clinical sign of relapse before FDG-PET examination and all six had refractory disease confirmed by biopsy directed to FDG-avid foci. As concerns the two patients who relapsed despite negative end-of-treatment PET, failure was identified at 31 months and at 68 months after treatment [22]. Different reasons might be raised for the superior sensitivity of end-of-treatment PET. Some tumors have initial response to chemotherapy but refractory clones might be selected and show late resurgence during the course of treatment [23]. Also, interim PET is performed shortly after a chemotherapy cycle (e.g., 10 days after the 2 nd cycle). With this short timing, there might be a "stunning effect". Our study pointed out that interim-PET has a lower sensitivity than end-of-treatment PET ( $46.7 \%$ vs $80 \%$; $P=0.0078$ ) so that a negative interim-PET study cannot obviate the need for end-PET examination [22]. Data from larger studies are now needed in order to determine more precisely what percentage of those patients with negative-interim PET and treatment failure can be depicted by end-of-treatment PET evaluation.

Unfortunately, end-of-treatment PET was not mandatory in the RATHL trial [14] and results are not available in the
SWOG S0816 trial [13], so we do not know how many relapses could have been predicted. It would be very helpful to know how many of the 58 patients with interim PET-negative patients and treatment failure in the SWOG S0816 trial had a positive end-of-chemotherapy PET [13]. In the ongoing GITIL HD0607 trial and in the HD0801 trial [24], patients who were negative at interim PET received end-of-treatment PET examination and, if negative, were randomized to radiotherapy vs no radiotherapy. Final results are not yet available. Additionally, these prospective studies could be of importance to define the optimal criteria for end-of-treatment PET positivity ( $>$ mediastinal blood pool, or $>$ liver).

Despite limited sensitivity, interim-PET is of value, especially so in advanced HL. About 30 to $35 \%$ of ABVD-treated advanced HL patients experience therapy failure. Early identification of some of these patients with resistance to therapy is an important issue.

Some studies are exploring the combination of interimPET results with additional parameters derived from baseline imaging [25], or from lymphoma tissue analysis and biological biomarkers [26-29]. One team showed that textural analysis of baseline $\mathrm{CT}$ images are correlated to progression-free survival and can be combined with information from interim-PET [30]. There also is potential for textural analysis of baseline PET images to yield useful information [31, 32]. Numerous biomarkers within the Reed-Sternberg cell and its microenvironment can also be exploited [33]. Agostinelli and colleagues recently analyzed the prognostic value of a set of three immunochemistry markers: STAT1, CD68 and PD-1 [27]. Following a regression tree analysis of a training set of patients, they successfully individualized a high-risk group that predicts failure within interim PET-negative patients. However, results were not fully confirmed in the validation set as the number of patients with negative interim-PET but high risk biological markers was quite limited, not allowing statistical analysis.

In conclusion, until further results from completed and ongoing trials are available, we suggest that end-ofchemotherapy PET assessment be maintained in interim PET-negative patients with advanced Hodgkin's lymphoma in order to identify patients who require radiation therapy on a residual active mass or immediate salvage therapy.

\section{Electronic supplementary material}

Funding No funding.

\section{Conflict of interest None.}

Ethical approval This article does not contain any studies with human participants or animals performed by any of the authors. 


\section{References}

1. Canellos GP, Rosenberg SA, Friedberg JW, Lister TA, Devita VT. Treatment of Hodgkin lymphoma: a 50-year perspective. J Clin Oncol. 2014;32:163-8.

2. Ansell SM. Hodgkin lymphoma: 2016 update on diagnosis, riskstratification, and management. Am J Hematol. 2016;91:434-42.

3. Cheson BD, Fisher RI, Barrington SF, Cavalli F, Schwartz LH, Zucca E, et al. Recommendations for initial evaluation, staging, and response assessment of Hodgkin and non-Hodgkin lymphoma: the Lugano classification. J Clin Oncol. 2014;32:3059-68.

4. Eichenauer DA, Engert A, Andre M, Federico M, Illidge T, Hutchings M, et al. Hodgkin's lymphoma: ESMO clinical practice guidelines for diagnosis, treatment and follow-up. Ann Oncol. 2014;25(Suppl 3):iii70-5.

5. Moulin-Romsee G, Hindie E, Cuenca X, Brice P, Decaudin D, Benamor M, et al. (18)F-FDG PET/CT bone/bone marrow findings in Hodgkin's lymphoma may circumvent the use of bone marrow trephine biopsy at diagnosis staging. Eur J Nucl Med Mol Imaging. 2010;37:1095-105.

6. El-Galaly TC, d'Amore F, Mylam KJ, de Nully BP, Bogsted M, Bukh A, et al. Routine bone marrow biopsy has little or no therapeutic consequence for positron emission tomography/computed tomography-staged treatment-naive patients with Hodgkin lymphoma. J Clin Oncol. 2012;30:4508-14.

7. Barrington SF, Kirkwood AA, Franceschetto A, Fulham MJ, Roberts TH, Almquist H, et al. PET-CT for staging and early response: results from the response-adapted therapy in advanced Hodgkin lymphoma study. Blood. 2016;127:1531-8.

8. Spaepen K, Stroobants S, Dupont P, Thomas J, Vandenberghe P, Balzarini J, et al. Can positron emission tomography with [(18)F]fluorodeoxyglucose after first-line treatment distinguish Hodgkin's disease patients who need additional therapy from others in whom additional therapy would mean avoidable toxicity? Br J Haematol. 2001;115:272-8

9. Weihrauch MR, Re D, Scheidhauer K, Ansen S, Dietlein M, Bischoff S, et al. Thoracic positron emission tomography using $18 \mathrm{~F}$-fluorodeoxyglucose for the evaluation of residual mediastinal Hodgkin disease. Blood. 2001;98:2930-4.

10. Terasawa T, Nihashi T, Hotta T, Nagai H. 18F-FDG PET for posttherapy assessment of Hodgkin's disease and aggressive nonHodgkin's lymphoma: a systematic review. J Nucl Med. 2008;49: $13-21$.

11. Adams HJ, Nievelstein RA, Kwee TC. Outcome of Hodgkin lymphoma patients with a posttreatment (18)F-Fluoro-2-deoxy-d-glucose positron emission tomography (FDG-PET)-negative residual mass: systematic review and meta-analysis. Pediatr Hematol Oncol. 2015;32:515-24.

12. Cheson BD, Pfistner B, Juweid ME, Gascoyne RD, Specht L, Horning SJ, et al. Revised response criteria for malignant lymphoma. J Clin Oncol. 2007;25:579-86.

13. Press OW, Li H, Schoder H, Straus DJ, Moskowitz CH, LeBlanc $\mathrm{M}$, et al. US intergroup trial of response-adapted therapy for stage III to IV Hodgkin lymphoma using early interim Fluorodeoxyglucose-positron emission tomography imaging: southwest Oncology group S0816. J Clin Oncol. 2016;34:2020-7.

14. Johnson P, Federico M, Kirkwood A, Fossa A, Berkahn L, Carella A, et al. Adapted treatment guided by interim PET-CT scan in advanced Hodgkin's lymphoma. N Engl J Med. 2016;374:241929.

15. Coyle M, Kostakoglu L, Evens AM. The evolving role of responseadapted PET imaging in Hodgkin lymphoma. Ther Adv Hematol. 2016;7:108-25.

16. Gallamini A, Barrington SF, Biggi A, Chauvie S, Kostakoglu L, Gregianin $\mathrm{M}$, et al. The predictive role of interim positron emission tomography for Hodgkin lymphoma treatment outcome is confirmed using the interpretation criteria of the Deauville five-point scale. Haematologica. 2014;99:1107-13.

17. Merli F, Luminari S, Gobbi PG, Cascavilla N, Mammi C, Ilariucci F, et al. Long-term results of the HD2000 trial comparing ABVD versus BEACOPP versus COPP-EBV-CAD in untreated patients with advanced Hodgkin lymphoma: a study by Fondazione Italiana Linfomi. J Clin Oncol. 2016;34:1175-81.

18. Younes A, Ansell SM. Novel agents in the treatment of Hodgkin lymphoma: biological basis and clinical results. Semin Hematol. 2016;53:186-9.

19. Hutchings M, Loft A, Hansen M, Pedersen LM, Buhl T, Jurlander J, et al. FDG-PET after two cycles of chemotherapy predicts treatment failure and progression-free survival in Hodgkin lymphoma. Blood. 2006;107:52-9.

20. Strobel K, Schaefer NG, Renner C, Veit-Haibach P, Husarik D, Koma AY, et al. Cost-effective therapy remission assessment in lymphoma patients using 2-[fluorine-18]fluoro-2-deoxy-D-glucose-positron emission tomography/computed tomography: is an end of treatment exam necessary in all patients? Ann Oncol. 2007;18:658-64.

21. Zinzani PL, Rigacci L, Stefoni V, Broccoli A, Puccini B, Castagnoli A, et al. Early interim 18F-FDG PET in Hodgkin's lymphoma: evaluation on 304 patients. Eur J Nucl Med Mol Imaging. 2012;39:4-12.

22. Mesguich C, Cazeau AL, Bouabdallah K, Soubeyran P, Guyot M, Milpied N, et al. Hodgkin lymphoma: a negative interim-PET cannot circumvent the need for end-of-treatment-PET evaluation. Br J Haematol. 2016;175:652-60.

23. Fanti S, Castellucci P, Stefoni V, Nanni C, Tani M, Rubello D, et al. Early relapse in a patient with Hodgkin's disease and negative interim FDG-PET. Ann Nucl Med. 2008;22:429-32.

24. Zinzani PL, Broccoli A, Gioia DM, Castagnoli A, Ciccone G, Evangelista $\mathrm{A}$, et al. Interim positron emission tomography response-adapted therapy in advanced-stage Hodgkin lymphoma: final results of the phase II part of the HD0801 study. J Clin Oncol. 2016;34:1376-85.

25. Casasnovas O, Kanoun S, Tal I, Cottereau AS, Edeline V, Brice P, et al. Baseline total metabolic volume (TMTV) to predict the outcome of patients with advanced Hodgkin lymphoma (HL) enrolled in the AHL2011 LYSA trial. J Clin Oncol. 2016;34.

26. Cuccaro A, Annunziata S, Cupelli E, Martini M, Calcagni ML, Rufini V, et al. CD68+ cell count, early evaluation with PET and plasma TARC levels predict response in Hodgkin lymphoma. Cancer Med. 2016;5:398-406.

27. Agostinelli C, Gallamini A, Stracqualursi L, Agati P, Tripodo C, Fuligni F, et al. The combined role of biomarkers and interim PET scan in prediction of treatment outcome in classical Hodgkin's lymphoma: a retrospective, European, multicentre cohort study. Lancet Haematol. 2016;3:e467-e79.

28. Bari A, Marcheselli L, Marcheselli R, Pozzi S, Cox MC, Baldessari $\mathrm{C}$, et al. Absolute monocyte count at diagnosis could improve the prognostic role of early FDG-PET in classical Hodgkin lymphoma patients. Br J Haematol. 2016. doi:10.1111/bjh.14406.

29. Vandenberghe P, Wlodarska I, Tousseyn T, Dehaspe L, Dierickx D, Verheecke M, et al. Non-invasive detection of genomic imbalances in Hodgkin/reed-Sternberg cells in early and advanced stage Hodgkin's lymphoma by sequencing of circulating cell-free DNA: a technical proof-of-principle study. Lancet Haematol. 2015;2:e5565 .

30. Ganeshan B, Miles KA, Babikir S, Shortman R, Afaq A, Ardeshna $\mathrm{KM}$, et al. CT-based texture analysis potentially provides prognostic information complementary to interim fdg-pet for patients with hodgkin's and aggressive non-hodgkin's lymphomas. Eur Radiol. 2017;27:1012-20. 
31. Hatt M, Majdoub M, Vallieres M, Tixier F, Le Rest CC, Groheux D, et al. 18F-FDG PET uptake characterization through texture analysis: investigating the complementary nature of heterogeneity and functional tumor volume in a multi-cancer site patient cohort. J Nucl Med. 2015;56:38-44.
32. Lartizien C, Rogez M, Niaf E, Ricard F. Computer-aided staging of lymphoma patients with FDG PET/CT imaging based on textural information. IEEE J Biomed Health Inform. 2014;18:946-55.

33. Scott DW, Steidl C. The classical Hodgkin lymphoma tumor microenvironment: macrophages and gene expression-based modeling. Hematology Am Soc Hematol Educ Program. 2014;2014:144-50. 